\title{
Charles-Gaspard de la Rive (1770-1834) médecin aliéniste et physicien
}

Par Isaac Benguigui

Pendant des siècles, les aliénés étaient considérés comme des possédés du démon et traités en conséquence, c'est-à-dire par les exorcismes et par les bûchers. Plus tard, on les assimila aux pires malfaiteurs. On les tenait alors enchaînés dans les plus affreux cachots. Jusqu'au XVIII ${ }^{\mathrm{e}}$ siècle, les malades mentaux ne pouvant plus être assurés par leur famille, trouvent asile dans les diverses fondations charitables, ainsi qu'en prison.

On croyait encore que pour guérir la folie il fallait lutter de logique avec les malades. Il fallait avant tout briser la volonté du malade et le premier principe des asiles était celui de l'autorité, qui se traduisait par les punitions, les douches, l'isolement et les pires barbaries. A Genève, comme ailleurs, les aliénés étaient entassés et livrés à la brutalité de leurs gardiens. Cette situation décida Charles-Gaspard de la Rive de poursuivre la réforme engagée par un autre médecin genevois, Abraham Joly (1748-1812) qui supprima les chaînes et autres moyens de répression. Il faut attendre la fin du XVIII ${ }^{\mathrm{e}}$ siècle pour entendre les voix des Chiarugi, des Tuke, des Daquin, des Pinel, pour que l'aliéné soit considéré comme un malade et soigné dans un établissement spécial. Pour Gaspard de la Rive, l'aliéné doit être séparé du criminel et du déviant social. Il réclame et obtient la construction d'un asile pour une utilisation thérapeutique du séjour des malades.

Charles-Gaspard de la Rive est né à Genève le 14 mars 1770. Il était le deuxième fils d'Ami-Jean de la Rive et de Jeanne-Elisabeth Sellon. Il fit ses études au Collège de Genève et il eut pour précepteur, pendant plusieurs années de sa jeunesse, le célèbre criminaliste Etienne Dumont (1759-1829) qui influencera sa carrière ${ }^{1}$.

Il venait de commencer ses études de droit à l'Académie, lorsqu'il fut condamné par le Tribunal révolutionnaire et jeté en prison avec quelques amis, pendant les troubles politiques qui secouèrent Genève en 1794. Après quelques mois de détention, il fut lui et son ami, Alexandre Marcet (1770-1822), parmi ceux qui étaient bannis de Genève et qui ont pris le chemin d'Edimbourg ${ }^{2}$. William Cullen était mort en 1790 et Joseph Black (1728-1799) occupait encore la chaire de médecine et de chimie. Les aspects chimiques de la médecine avaient retenu l'attention de l'Université. Après 
trois années d'études, de la Rive obtint son titre de docteur en médecine. Son sujet de thèse était «Tentamen physiologicum de calore animali» (1797) où il exposa sa théorie sur la chaleur animale. Cette thèse est une critique d'Alain Crawford, dans laquelle de la Rive suit son maître John Allen en attibuant la chaleur animale à la combustion dans le sang de particules dérivées de la nourriture et de l'oxygène absorbé à travers les poumons. Il envisageait l'application des principes de la physique à l'explication du phénomène physiologique de la chaleur animale. Elle était dédicacée au médecin Charles Congalton et à Daniel Rutherford, professeur de botanique. Il passa une quatrième année à Edimbourg et devint président de la Royal Medical Society où il a lu une communication: "Does the theory of Aepinus account in a satisfactory manner for the principal phenomena of magnetism?" 3

Avant de rentrer à Genève, il consacra un voyage de plusieurs mois à visiter les principaux asiles d'aliénés d'Angleterre, entre autres, les célèbres établissements du Dr. Willis à Greatford et celui du Dr. Thomas Arnold à Leicester. Il adressa le $1^{\text {er }}$ juillet 1798, aux rédacteurs de la Bibliothèque Britannique, une lettre dans laquelle il donne une description de cet établissement, qui en révéla l'existence à Pinel et qui est restée classique ${ }^{4}$.

Après avoir classé les différentes formes de l'aliénation de l'esprit en trois genres: «la manie (ou folie proprement dite), la mélancolie et l'hypochondrie», trois maladies qui «se confondent souvent et quelquefois se succèdent les unes aux autres», de la Rive définit également la mélancolie comme une maladie idiopathique qui est souvent due à une «idée dominante» qui s'empare de l'esprit, borne ou diminue l'impression que les objets extérieurs devraient faire sur lui. Pour guérir le malade, il faut donc détruire cette «idée dominante». De la Rive insiste sur l'importance qu'il y a de transporter le malade hors de chez lui et d'éviter avec soin de lui présenter les objets qui peuvent lui rappeler «les idées absurdes qui constituent son mal». L'aliéniste genevois décrit les avantages de l'isolement et les circonstances qui contribuent à guérir les aliénés, quand ils sont transportés hors de chez eux et se voient entourés de personnes étrangères. Il recommande vivement de renoncer à la violence, «il faut éviter avec soin tous les moyens de terreur qui ne sont jamais exempts de danger» et de lui substituer un «système doux et modéré qui fait que le malade sent continuellement la contrainte». Il insiste également sur la relation de confiance qui doit s'établir entre le médecin et le malade, «ne jamais tromper les malades sur leur état». On ne s'étonnera pas après cette lettre de la réforme que de la Rive engagea à son retour à Genève (1799). C'est le début de l'ère asilaire. 
Nommé professeur honoraire de chimie pharmaceutique, il sera chargé en 1802 du soin des aliénés qui étaient alors placés à une dépendance de l'hôpital, nommée la «Discipline» ${ }^{5}$ (devenue aujourd'hui la prison de SaintAntoine). Cet établissement était construit en 1709, servait de maison de correction et les fous y étaient enfermés et enchaînés, abandonnés complètement à leurs gardiens. Dans ces locaux «défectueux, insalubres et exigus», il poursuivra la réforme engagée par Joly. De la Rive améliora considérablement la condition de ces infortunés. Il supprima tous les instruments de torture, les bains de surprise et autres cruelles inventions d'une thérapeutique ignorante et barbare.

Il signale à diverses reprises les inconvénients de la «Discipline» et la nécessité d'un nouvel établissement pour les aliénés. Ceux-ci sont désormais considérés comme des malades qu'il faut soigner et non garder en détention. «Le fou est un malade et non un coupable.» ${ }^{6}$. Le statut de l'aliéné change: il est élevé à la dignité de malade et il est soigné comme tel.

Un peu partout en Europe, différentes thérapeutiques de l'aliénation mentale sont proposées. A la fin du $\mathrm{XVIII}^{\mathrm{e}}$ siècle et au début du XIX ${ }^{\mathrm{e}}$, ce qu'on appelait le traitement moral de la folie est à l'ordre du jour. A ses débuts, il procède de la seule philanthropie qui, depuis le début des années 1780, veut rompre avec la barbarie des traitements antérieurs et, au-delà de leur caractère humanitaire, les soins moraux auront au moins pour premier résultat de ne pas mettre d'obstacle aux guérisons naturelles. Ainsi est fondée en 1796 «La Retraite» , par le philanthrope Quaker William Tuke (1732-1822) qui incarne les vertus Quaker de dignité humaine et de selfcontrôle. L'accent est mis sur la relation morale. Ce regard nouveau sur la folie est partagé par plusieurs médecins. Joseph Daquin (1732-1815), médecin à Chambery voit dans la folie une des maladies qui exige «le plus petit nombre de remèdes de la pharmacie» et recommande «la patience, beaucoup de douceur, une prudence éclairée» ${ }^{8}$. V. Chiarugi à Florence, Cullen en Angleterre et J.Th. Held à Prague, étaient également révoltés par les méthodes utilisées. L'électricité qui est appliquée au milieu du XVIII ${ }^{\mathrm{e}}$ siècle comme moyen thérapeutique dans de nombreuses maladies ${ }^{9}$, est rapidement préconisée dans le traitement de la folie où l'on pense qu'elle peut «détruire l'obstacle qui tient la raison et les sens enchaînés» (Daquin, $1792)^{10}$.

Dans ce courant d'idées, la nécessité d'un lieu de séjour réservé aux malades s'impose peu à peu. A cet effet, Charles-Gaspard de la Rive fut un des premiers auteurs à s'occuper de la statistique des aliénés. Il publia un 
mémoire remarquable pour l'époque ${ }^{11}$, dans lequel il étudia le rapport qui existe entre la population d'un pays et le nombre d'aliénés que donne en moyenne cette population. Il se demandait si «le nombre proportionnel des aliénés, relativement à la population, a augmenté d'une manière sensible ces derniers temps?» (Certains médecins prétendaient qu'il avait triplé entre 1810 et 1830.) Ce mémoire éclaira l'opinion sur ce sujet, au moment où l'on s'occupait à Genève d'un changement dans l'hospice des aliénés, qui était demandé depuis plusieures années. L'ancienne «Discipline» allait bientôt disparaître. En 1817, aidé par son ami Etienne Dumont, il avait proposé de transporter les aliénés à la campagne, pour améliorer leur sort, mais les circonstances financières n'étaient pas favorables à cette proposition et les emplacements offerts ne convenaient pas. Il faudra attendre 1832 pour voir son projet réalisé. L'épidémie du choléra qui gagna toute l'Europe cette année-ci obligea les responsables à débarrasser d'urgence la «Discipline» pour y mettre les cholériques ${ }^{12}$. Les aliénés furent transportés à la campagne, à Corsier, près de Genève, le 27 avril 1832. Ils y séjourneront jusqu'en $1838^{13}$. De la Rive s'efforça d'éviter le retour de ses patients à la «Discipline». «A aucun prix, dit-il, il ne faut permettre la réintégration de ces malheureux à la «Discipline». Cette mesure serait fatale pour nos malades qui changeraient un bon air, de vastes cours, de grands jardins et de jolies chambres contre leur triste prison, leur cour étroite avec ses hautes murailles froides, humides, malsaines...»

Pour de la Rive, un des principaux moyens de guérison dépend du local où les malades sont traités. Ce local doit contenir des moyens de promenades, d'exercices, pour donner la possibilité à presque tous les malades (les furieux exceptés) de sortir de leurs cellules et se promener en plein air; il faut en outre que les dimensions de ce local permettent une variété de travaux, particulièrement ceux du jardinage et de l'agriculture et cela en vue de la guérison. «Les occupations rurales et le train d'une ferme, étoient une salutaire distraction à la portée de tous et qui convient [aux malades] en général fort bien.» ${ }^{14}$ De la Rive observe que les manies étaient plus fréquentes parmi les habitants des villes que parmi ceux de la campagne. Il constate quelque rapport 'entre le degré de civilisation d'un pays et le nombre de cas de maladies mentales qu'on y découvre, il met en évidence l'augmentation des richesses comme un des facteurs favorable à cette maladie. Il pose également le problème de l'activité professionnelle: «Toute transition subite d'un état de grande agitation mentale à un état de complète oisiveté, est une cause fortement prédisposante d'un dérangement de l'esprit.» Il conclut que 
l'ennui après une vie occupée, surtout chez les personnes incapables de se procurer des moyens d'activité mentale, est une cause fréquente d'aliénation et conseille en conséquence de «faire germer chez les jeunes un goût quelconque, littéraire, scientifique, mécanique même, de peur que dans les moments de vide, ils deviennent la proie à ces funestes maladies de l'esprit». ${ }^{15}$ Cette thérapeutique active, celle du travail, préconisée par de la Rive, s'ajoute à la thérapeutique passive, celle de l'enfermement. Elle s'inscrit dans le cadre des autres moyens utilisés par les thérapeutes du XIX ${ }^{\mathbf{e}}$ siècle $^{16}$.

En 1828, Gaspard de la Rive fera un voyage à Londres et ramènera un plan d'un hospice conçu sur le modèle de Wakefield et qui était destiné à recevoir les aliénés de Londres. Ce plan fut mené sous la direction de Samuel Tuke (1784-1857). L'architecte genevois Vaucher fut chargé d'adapter le modèle anglais aux exigences genevoises (d'une capacité totale estimée à une soixantaine de malades - une moitié réservée aux hommes, une autre aux femmes). La tâche de Charles-Gaspard de la Rive désormais est consacrée à la création d'un asile «qui soit le résultat de toutes les lumières acquises sur cet objet». Ainsi, le $1^{\text {er }}$ juin 1838, cinquante-sept malades sont transférés à l'hospice des Vernaies (ou des Vernets). C'est le premier du genre en Suisse dans sa conception de lieu de séjour et de soins. L'asile des Vernaies abritera les aliénés de Genève de 1838 à $1900^{17}$. A partir de cette date, les malades sont alors transférés à l'hôpital de Bel-Air. De la Rive était mort le 18 mars 1834 et c'est le docteur Jean-Charles Coindet (1796-1876) qui lui succéda.

En médecine, il faut encore mentionner son étude comparative du sang en état de santé et de maladie ${ }^{18}$, et ses recherches sur l'emploi de l'acide nitreux dans le cas de la syphilis. De la Rive assure que cette maladie traitée avec de l'acide nitreux donne de bons résultats. Il précise: «Ce n'est pas l'acide nitrique concentré, couleur de paille qu'on emploie, mais bien l'acide nitreux fumant, tel qu'il est retiré après la première distillation du nitre avec l'acide sulfurique» ${ }^{19}$. Poursuivant ses travaux sur la chaleur animale, il montra que l'action galvanique avait quelque analogie avec la production de la chaleur animale. «Dans l'appareil voltaïque, la production de la chaleur, s'arrête lorsque l'eau a perdu par l'oxydation et la combinaison une partie de son acide ou de son oxygène; rendez à cette eau son acide ou son corps oxygéné, et recommencez l'opération, la chaleur reparaîtra, et cela tant qu'il restera du métal à oxyder. Dans l'animal, empêchez le sang de s'oxyder à la surface du poumon, ou par la ligature de l'aorte, mettez un obstacle à ce que le sang oxygéné arrive aux extrémités des artères, vous anéantissez la production de la chaleur animale.» ${ }^{20}$ 
De la Rive considère également la respiration comme une espèce de combustion et trouve que la température propre des différents animaux est à peu près proportionnelle à la quantité d'air qu'ils font passer par leurs poumons dans un temps donné.

De la Rive n'a pas seulement été un médecin aliéniste, mais il fut également un grand physicien et chimiste. Dès son retour à Genève, il se voua d'une manière toute particulière à ces deux sciences. C'est lui qui fit principalement connaître dans la Bibliothèque Britannique, les grandes découvertes scientifiques dont l'Angleterre s'enrichissait alors et particulièrement celles de Davy sur les effets si remarquables de la pile voltaïque. Les traductions, les résumés qu'il donnait de ces travaux, les notes et commentaires dont il les accompagnait étaient fort appréciés par les savants et étaient intégralement reproduits dans les Annales de chimie (Berthollet [1748-1822] était le rédacteur).

Les recherches que nous poursuivons sur les travaux de Gaspard de la Rive en chimie et physique feront l'objet d'un autre travail. Nous mentionnons pour le moment quelques-uns de ces travaux relatifs à la conversion de l'amidon en sucre, à l'analyse de la théorie des proportions chimiques et l'électrochimie de Berzelius, au traitement des métaux précieux, à la compositions du vin, aux effets du tremblement de terre du 19 février 1822 sur les eaux thermales d'Aix-en Savoie et aux changements qui en étaient résultés dans leur composition chimique. Son étude de l'arsenic et de sa présence dans des cas douteux d'empoisonnement permit d'obtenir par lavage à l'eau chaude, de filtration et d'évaporations successives, l'arsenic lui-même: «le sulfate de cuivre et la potasse ne garantissent pas la certitude de l'existence de l'arsenic. D'autres réactifs peuvent donner la même couleur (précipités d'un bleu foncé)». ${ }^{21}$ En physique nous mentionnons son mémoire sur les sons produits dans les tubes par la flamme d'un gaz d'hydrogène. Ses travaux en électricité sont d'une grande importance, en particulier l'électricité voltaïque. On lui doit la construction de la première pile sur le continent qui fut l'objet d'admiration de savants suisses et étrangers.

Sa correspondance avec les savants européens est d'une importance considérable, en particulier avec Ampère, Davy et Faraday ${ }^{22}$. En 1823, il introduit, auprès de ce dernier, le colonel Fischer de Schaffouse, décrit par de la Rive comme «l'inventeur de plusieurs procédés et méthodes nouvelles et ingénieuses pour la fabrication et le travail de l'acier»». ${ }^{23} \mathrm{Il}$ collabora d'une manière très active aux expériences de ces trois savants auxquelles il faut ajouter celles d'Arago ${ }^{24}$. 
En dehors de ses recherches et de son enseignement, il joua également un rôle dans la vie publique. Elu premier Syndic en 1817, il démissionna pour raison de santé en 1818 et reprit sa place de professeur. Il fut recteur de l'Académie de 1823 à 1825 où il donna une nouvelle orientation à l'enseignement scientifique. Il fut l'un des fondateurs les plus généreux de la Société de lecture, du Musée d'histoire naturelle et du Jardin botanique.

\section{Notes}

1 Auteur anonyme, Notice biographique sur M. le professeur G. de la Rive, in Bibliothèque universelle des sciences, belles lettres et arts, t. LV, 1834, pp.303-338. Cf. également la notice que lui a consacrée Jean Alfred Gautier, in Mémoires de la Société de physique et d'histoire, t. X, 1843, pp. XII-XIV.

${ }^{2}$ Charles-Gaspard de la Rive quittait Genève le 21 août 1794. «Je quittais ma malheureuse patrie que les grands principes d'heureux et d'agréables séjours avaient changé en un repaire de brigands». Cf. Journal, Msfr 2312, p. 14, Bibliothèque publique et universitaire (B.P. U.) de Genève.

${ }^{3}$ Les travaux d'Aepinus (1724-1802) ramenaient les phénomènes électriques et magnétiques à une même explication.

${ }^{4}$ Sur un nouvel établissement pour les guérisons des aliénés, in Bibliothèque Britannique, t. VIII, pp.300-327.

${ }^{5}$ La construction d'une maison de discipline entrait dans le plan général arrêté au commencement du XVIII ${ }^{e}$ siècle. Le 19 janvier 1709 , la Seigneurerie autorisa la direction de l'hôpital à bâtir sur un emplacement situé sur le boulevard Saint-Antoine, donné à l'hôpital en 1699.

${ }^{6}$ Starobinski, Jean, Histoire de la médecine, Lausanne, 1964, p. 64.

7 Une ferme était annexée à cet asile. De la Rive la visitera en 1798 «j'en fus vraiment édifié» dira-t-il.

${ }^{8}$ C'est en 1791 qu'il publiera La philosophie de la folie, où, pour la première fois, il exposera les principes de ce qui va devenir le traitement moral.

${ }^{9}$ Voir le chapitre consacré à l'électrothérapie dans notre ouvrage Théories électriques $d u$ XVIII I siècle, éd. Georg, Genève, 1984, pp. 53-78.

10 Nouvelle histoire de la psychiatrie, Toulouse, 1983, p. 436, sous la direction de Postel, Jacques et Quétel, Claude.

${ }^{11}$ Sur la statistique des affections mentales, in Bibliothèque Universelle, 1830, t.XLIII, pp. 1-24.

12 Morsier, Georges de, Histoire de la psychiatrie et de la neurologie à Genève, Gesnerus, 34, 1977, p. 188.

${ }^{13}$ De la Rive présenta un mémoire au Conseil d'Etat le 25 février 1833, intitulé Sur l'établissement des aliénés. L'inauguration des Vernaies eut lieu le 20 mai 1838. De la Rive était mort le 18 mars 1834 et le docteur Jean-Charles Coindet (1796-1876) lui succéda.

${ }^{14}$ On parlait alors de colonies agricoles et des asiles dits «aux portes ouvertes». Les premiers essais de colonies agricoles pour le traitement des aliénés remontent à 1832 et ont été faits en France, dans la ferme Sainte-Anne. En Suisse, le premier essai fut fait en 1848, dans le canton de Saint-Gall. 
15 Statistique des affections mentales, Bibliothèque Universelle, février 1830, p. 23.

${ }^{16}$ Starobinski, Jean, Histoire du traitement de la mélancolie des origines à 1900. Acta psychosomatica, Bâle (J. R. Geigy) 1960, pp.49-82.

${ }^{17}$ Ladame, P[aul]-L[ouis], Le Nouvel Asile des Aliénés à Genève, Genève, 1895 et Bouvier, Philippe, De l'asile à la cité, Genève, 1981, éd. Médecine et Hygiène.

${ }_{18}$ Bibliothèque Britannique, t. III, p. 337.

${ }^{19}$ Loc. cit., t. IV, p. 68.

${ }^{20}$ Observations sur les causes présumées de la chaleur propre des animaux, lues à la Société de physique et d'histoire naturelle de Genève, le 4 septembre 1820. In Bibliothèque Universelle, 1820, pp.103-111.

${ }^{21}$ Procédé recommandé par G. de la Rive pour constater la présence de l'arsenic, lorsqu'il est mêlé dans d'autres substances, Bibliothèque Britannique, t. XLI, p.172. Cet article mentionne le cas d'une dame de Lancastre qui mourut le 25 mars 1808 avec des symptômes qui semblaient annoncer qu'elle avait été empoisonnée par l'arsenic.

${ }^{22}$ Le résumé des lettres adressées par Ampère à Gaspard de la Rive (au nombre de cinq) est donné par Marc Cramer et Georges de Morsier sous le titre: L'importance des expériences faites à Genève par Gaspard et Auguste de la Rive pour la découverte de l'électromagnétisme, Gesnerus, 28, 1971, Fasc.3/4), pp.234-235.

${ }^{23}$ Tunbridge, Paul, Faraday's Genevese friends. Notes Records of the Royal Society of London, 1973, 27, p. 269.

${ }^{24}$ Arago qui a visité Genève en été 1820, a participé aux expériences menées par Gaspard de la Rive en présence de Prévost, Pictet, Th. de Saussure, Marcet et de Candolle, pour vérifier les résultats obtenus par Oersted concernant l'action exercée par un courant électrique sur l'aiguille aimantée.

De même Ampère qui visita Genève en 1822, fera quelques expériences sur le courant galvanique dans le laboratoire de Gaspard de la Rive. Le 24 septembre 1823, de la Rive informa Faraday du résultat de ces expériences. Faraday et Davy se sont rendus également à Genève en 1814 sur l'invitation de Gaspard de la Rive.

\section{Summary}

The author shows the action conducted by Gaspard de la Rive for the change of the lunatic status and the struggle he conducted for the building of an asylum in Geneva, and he mentions his very important studies in physics and chemistry.

Dr. Isaac Benguigui

L.D.E.S. Université de Genève

11, avenue des Cavaliers

CH-1224 Genève 